\title{
Comparative proteomic analysis of Salmonella enterica serovar Typhimurium ppGpp-deficient mutant to identify a novel virulence protein required for intracellular survival in macrophages
}

Takeshi Haneda', Mariko Sugimoto ${ }^{1}$, Yukie Yoshida-Ohta ${ }^{1}$, Yoshio Kodera ${ }^{2}$, Masamichi Oh-Ishi ${ }^{2}$, Tadakazu Maeda², Satomi Shimizu-Izumi ${ }^{1}$, Tsuyoshi Miki ${ }^{1}$, Yoshinori Kumagai ${ }^{1}$, Hirofumi Danbara ${ }^{1}$, Nobuhiko Okada ${ }^{1 *}$

\begin{abstract}
Background: The global ppGpp-mediated stringent response in pathogenic bacteria plays an important role in the pathogenesis of bacterial infections. In Salmonella enterica serovar Typhimurium (S. Typhimurium), several genes, including virulence genes, are regulated by $\mathrm{ppGpp}$ when bacteria are under the stringent response. To understand the control of virulence genes by ppGpp in S. Typhimurium, agarose 2-dimensional electrophoresis (2-DE) combined with mass spectrometry was used and a comprehensive 2-DE reference map of amino acid-starved S. Typhimurium strain SH100, a derivative of ATCC 14028, was established.

Results: Of the 366 examined spots, 269 proteins were successfully identified. The comparative analysis of the wild-type and $\mathrm{ppGpp}^{0}$ mutant strains revealed 55 proteins, the expression patterns of which were affected by ppGpp. Using a mouse infection model, we further identified a novel virulence-associated factor, STM3169, from the ppGpp-regulated and Salmonella-specific proteins. In addition, Salmonella strains carrying mutations in the gene encoding STM3169 showed growth defects and impaired growth within macrophage-like RAW264.7 cells. Furthermore, we found that expression of stm3169 was controlled by ppGpp and SsrB, a response regulator of the two-component system located on Salmonella pathogenicity island 2.
\end{abstract}

Conclusions: A proteomic approach using a 2-DE reference map can prove a powerful tool for analyzing virulence factors and the regulatory network involved in Salmonella pathogenesis. Our results also provide evidence of a global response mediated by ppGpp in S. enterica.

\section{Background}

The facultative intracellular bacterium Salmonella enterica causes a broad spectrum of diseases, such as gastroenteritis and bacteremia, which are typically acquired by oral ingestion of contaminated food or water. S. enterica serovar Typhimurium ( $S$. Typhimurium) causes enterocolitis in humans and a typhoid-like systemic infection in mice.

Several virulence genes associated with Salmonella pathogenicity islands (SPIs) and the virulence plasmid have been characterized in $S$. Typhimurium. Two type

\footnotetext{
* Correspondence: okadan@pharm.kitasato-u.ac.jp

'Department of Microbiology, School of Pharmacy, Kitasato University, 5-9-1

Shirokane, Minato-ku, Tokyo 108-8641, Japan

Full list of author information is available at the end of the article
}

III secretion systems (T3SS) encoded by SPI-1 and SPI-2 play central roles in Salmonella pathogenesis. SPI1 is essential for the invasion of host cells and the induction of apoptosis in infected macrophages [1,2]. SPI-2 T3SS primarily confers survival and replication on macrophages and is required for systemic infection in the mouse infection model [3,4]. Expression of SPI-2 genes is induced within a modified phagosome, called the Salmonella-containing vacuole (SCV), in infected macrophages [5]. Induction of SPI-2 genes depends on a two-component regulatory system, SsrA/SsrB, encoded within the SPI-2 region [6]. Expression of SsrAB is also mediated by two-component regulatory systems, OmpR/ EnvZ and PhoP/PhoQ, which sense osmotic stress and cation limitation, respectively $[7,8]$. In addition, a global

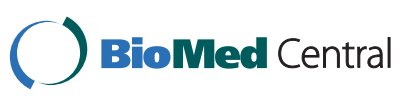

(c) 2010 Haneda et al; licensee BioMed Central Ltd. This is an Open Access article distributed under the terms of the Creative Commons Attribution License (http://creativecommons.org/licenses/by/2.0), which permits unrestricted use, distribution, and reproduction in any medium, provided the original work is properly cited. 
transcriptional regulator, SlyA, which interacts directly with the $s s r A$ promoter region, is involved in the expression of SPI-2 T3SS [9-11].

During infection of mammalian hosts, $S$. Typhimurium has to rapidly adapt to different environmental conditions encountered in its passage through the gastrointestinal tract and its subsequent uptake into epithelial cells and macrophages. Thus, establishment of infection within a host requires coordinated expression of a large number of virulence genes necessary for the adaptation between extracellular and intracellular phases of infection. It has been demonstrated that the stringent response plays an important role in the expression of Salmonella virulence genes during infection [12-14].

The stringent response is mediated by the signal molecules, guanosine tetraphosphate (ppGpp) and guanosine pentaphosphate (pppGpp) (both are referred to as ppGpp in this manuscript), which accumulate in bacterial cells and exert both positive and negative effects on the transcription of many genes. ppGpp plays an important role in the virulence of pathogenic bacteria [15]. In Gram-negative bacteria, ppGpp is synthesized by two tynthases, the synthase I and the synthase II, which are encoded by the relA and spoT genes, respectively [16]. These enzymes respond differently to environmental conditions. RelA is activated by the binding of uncharged tRNA to ribosomes upon amino acid starvation. SpoT is induced during the exponential growth phase and responds to other changes in environmental conditions, specifically a lack of carbon sources or energy deprivation [17]. ppGpp binds directly to the $\beta$ and $\beta$ ' subunits of RNA polymerase (RNAP), leading to destabilization of the RNAP-rRNA promoter open complex [18]. Moreover, the stringent response is increased by the availability of free RNAP, which gives rise to $\sigma$ competition [19]. ppGpp indirectly activates the expression of many stress-induced genes by its release from RNAP $\sigma^{70}$-dependent promoters and by facilitating the use of alternative $\sigma$ factors. It has been shown that ppGpp is not only essential for surviving periods of stress but also for the interaction of bacteria with their host [20].

In case of $S$. Typhimurium, a mutant strain deficient in both relA and spoT $(\Delta$ relA $\Delta$ spoT) shows marked reductions in both bacterial invasion into host cells and proliferation in macrophages [12,13]. Furthermore, the virulence of the $\Delta$ relA $\Delta$ spoT mutant is severely attenuated in mice $[12,13]$. ppGpp controls the expression of SPI-1 to -5 and Spv through their transcriptional regulators HilA, InvF, RtsA, SsrA, SlyA, and SpvR [12-14,21]. These observations indicate that ppGpp may play a major role in Salmonella virulence via the altered expression of regulatory genes. Because ppGpp has been shown to affect the expression of many virulence genes in $S$. Typhimurium, it is likely that there are additional virulence genes among the ppGpp-regulated genes.

In this study, we constructed an agarose 2-dimensional electrophoresis (2-DE) reference map of $S$. Typhimurium grown under amino acid starvation to identify ppGpp-regulated proteins from whole-cell preparations. By comparative proteomic analysis of ppGpp-regulated and Salmonella-specific proteins, we identified a novel virulence factor, STM3169, required for intracellular survival within macrophages.

\section{Results and Discussion}

\section{Agarose 2-DE reference map of $S$. Typhimurium with induced stringent responses}

Because the correlation between mRNA and protein expression levels is nonpredictive, the direct measurement of protein expression is essential for the analysis of biological processes [22]. 2-DE allows several hundred proteins to be displayed on a single gel, thus producing a direct and global view of the proteome at a given time point [23]. Agarose 2-DE takes advantage of the process of protein separation over a broad range $[24,25]$. In this study, to separate and identify more proteins, we applied agarose 2-DE to the bacterial proteome, and also used $12 \%$ and $15 \%$ SDS-PAGE gels for the second dimension. Whole-cell proteins were obtained from the $S$. Typhimurium strain SH100, a derivative of ATCC 14028, with the stringent response induced by serine hydroxamate, as described previously [26]. Agarose 2-DE was performed at least three times on independent samples. More than 350 protein spots from the strain were detected on each 2-DE gel stained with Coomassie Brilliant Blue. To identify proteins on the agarose 2-DE gels, we excised 230 spots from the $12 \%$ gel and 136 spots from the $15 \%$ gel. We finally identified a total of 360 proteins (273 proteins from the $12 \%$ gel [Figure 1A] and 87 proteins from the $15 \%$ gel [Figure 1B]) by MS/ MS analysis out of 307 protein spots (232 spots from the $12 \%$ gel and 75 spots from the $15 \%$ gel) that were successfully excised (see additional file: 1). In total, 267 proteins were obtained from the gels, with 40 proteins identified as being redundant. The highest and lowest molecular masses of identified proteins were $93.4 \mathrm{kDa}$ for AcnB (aconitate hydrase 2, spot 188) and $7.4 \mathrm{kDa}$ for CspC (cold-shock protein, spot 303), respectively. Fifty spots (35 spots from the $12 \%$ gel and 15 spots from the $15 \%$ gel) were found in a basic range. Interestingly, 78 protein spots $(25.4 \%)$ were annotated as putative proteins on the genome of the $S$. Typhimurium LT2 strain, which is more than $98 \%$ identical in sequence to the 14028 strain [27].

We estimated the molecular weight of the protein spots on the 2-DE gels and compared them with the theoretical molecular weight of strain SH100. While 


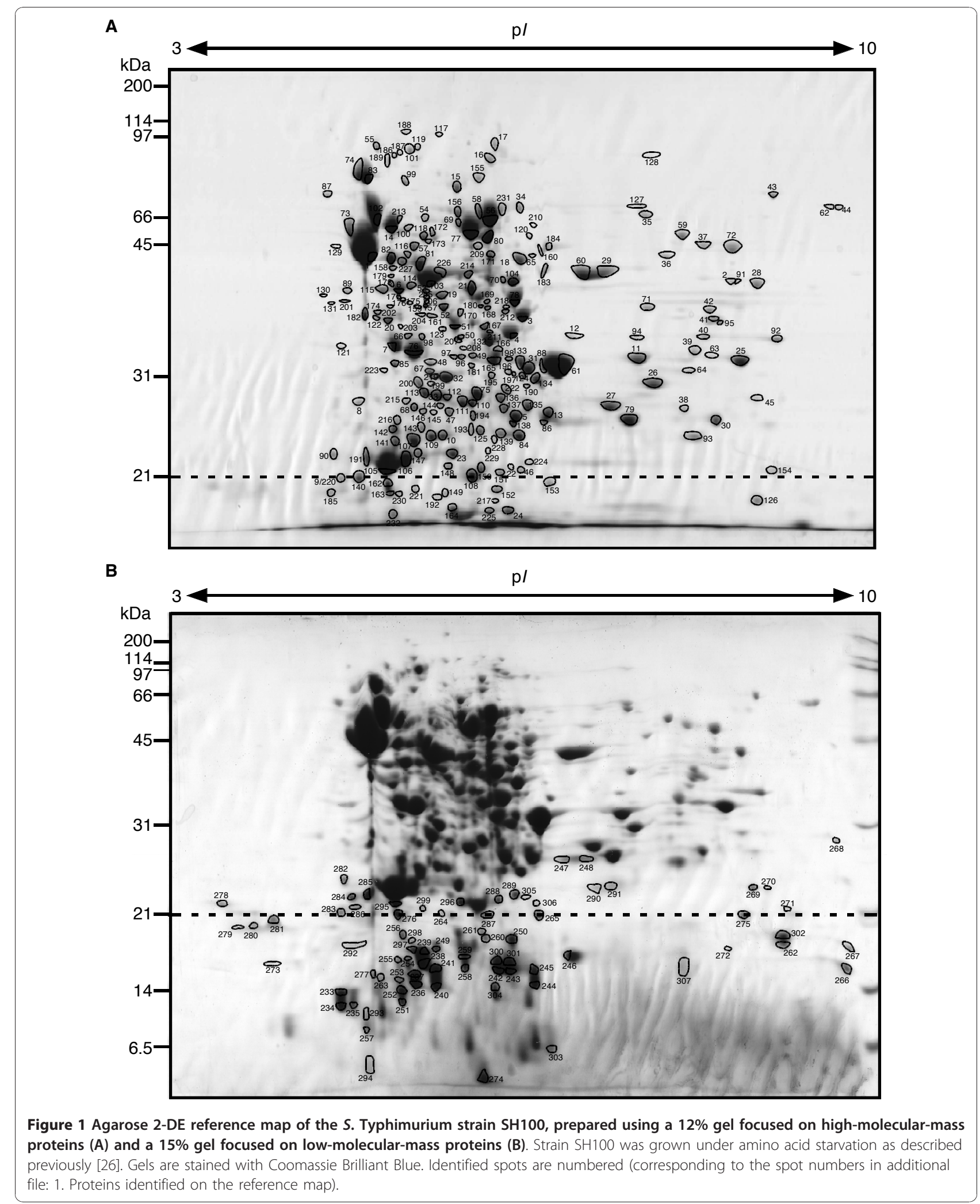


most of the estimated molecular weight values matched the theoretical values, we found 14 protein spots on the map that had different experimental and predicted molecular weights values (Figure 2). These proteins might be post-translationally modified by proteolytic processing, phosporylatoin of multiple amino acid residues and/or an artifact caused by sample preparation. For example, the experimental molecular weight of OmpA indicated that the protein was likely processed by a proteolytic enzyme, because two different spots (spot nos. 152 and 287) were identified as OmpA, the experimental masses of which were significantly lower than the theoretical values. Similar results were described in other reports [28,29].

Next, we classified proteins identified on the map using the KEGG pathway database. While 156 proteins (45.3\%) were classified into several metabolic categories (carbohydrate, energy, lipid, nucleotide, amino acid, and other amino acids), 70 proteins (22.8\%) were grouped in the no entry category, which means that these proteins do not belong to the other categories. This category contained 20 known virulence-associated proteins, including flagella and flagella biosynthesis proteins (FliC, FljB, FliY, FliG, FliM, and FliD), SPI-1 effectors (SipD, SopB, and SopE2), an SPI-1 translocase (SipC), an iron transporter (SitA), superoxide dismutases (SodA, SodB, SodC1, and SodC2), a quorum-sensing protein (LuxS), a two-component response regulator (PhoP), peptidyl-

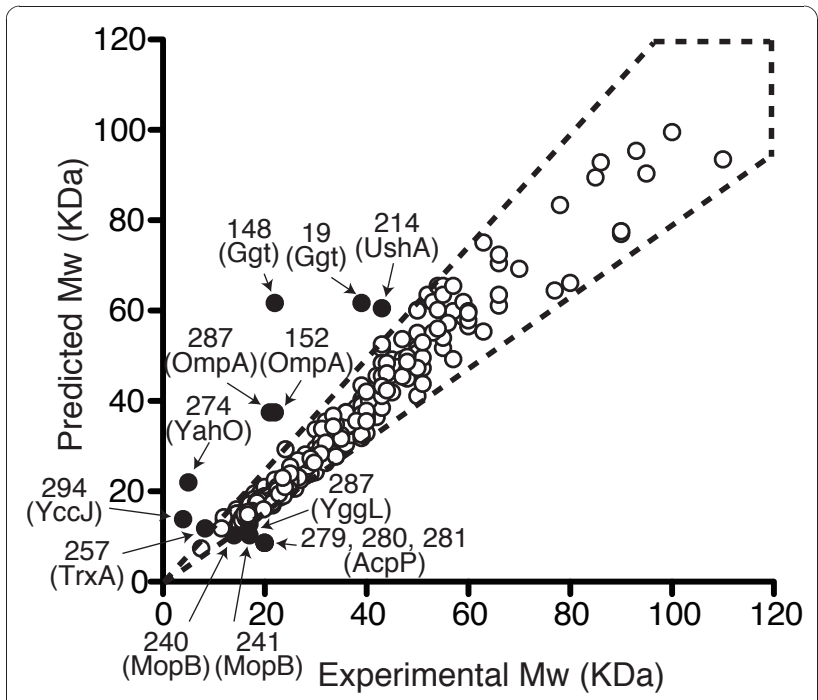

Figure 2 Comparison of the gel-estimated and theoretically calculated molecular weight (Mw) of the identified protein spots. Arrows indicate spot numbers and identified proteins, which have different Mw between the experimental and the predicted ones. The proteins in the area enclosed by the dotted lines denote that they have an experimental Mw within $\pm 25 \%$ of the predicted molecular mass. prolyl cis-trans isomerases (FkpA and SurA), and a periplasmic disulfide isomerase (DsbA).

\section{Identification of ppGpp-regulated proteins using comparative proteomics}

To identify proteins associated with the stringent response in $S$. Typhimurium, we compared the agarose 2 -DE pattern for each total protein prepared from amino acid-starved $S$. Typhimurium SH100 and $\Delta$ relAsspoT strain (TM157) (Figure 3). As shown in Table 1, 24 protein spots (23 proteins) were found at higher levels in SH100 than in TM157, while 23 protein spots were found at lower levels in SH100 than in TM157. We focused on 23 proteins, which were positively regulated by ppGpp in the stringent response.

Of these proteins, six genes (treA, ugpB, ynhG, yliB, $u g p B, \operatorname{deg} Q)$ had previously been identified as ppGppregulated genes in $E$. coli at the transcriptional level [30]. In $S$. Typhimurium, it has been shown that ppGpp controls the expression of known virulence-associated genes, including $\operatorname{sip} C, f l i Y, \operatorname{sop} B$, and $\operatorname{sod} C 1$, in response to growth conditions relevant to host infection [14]. Thus, to confirm the results from the comparative proteomic analysis, mRNA levels of the remaining 13 genes were assessed by qRT-PCR. As a result, mRNA expression levels of eight genes (stm3169, cpdB, tolB, ydgH, $o p p A, y a j Q, y h b N, y t f J)$ were significantly higher in SH100 than in TM157 under stringent conditions (Table 1).

\section{Identification of novel virulence-associated factors regulated by ppGpp}

Among 13 genes newly identified as ppGpp regulated, 12 genes were present in non-pathogenic E. coli $\mathrm{K}-12$ strain. Therefore, to examine whether ppGpp-regulated putative or hypothetical proteins could contribute to the virulence of $S$. Typhimurium, we chose Salmonellaspecific protein, STM3169, which is present in S. Typhimurium, but is absent in the E. coli $\mathrm{K}-12$ strain (Figure 4 $[27,31])$. To determine the roles of STM3169 in virulence, a deletion mutant was constructed in the $S$. Typhimurium wild-type SH100 strain, and its virulence was assessed by a mouse mixed infection using a competitive index analysis. As shown in Figure 5A, mouse mixed infections showed that disruption of the stm3169 gene conferred a defect in virulence in mice, and that successful complementation was achieved for TH973 ( $\Delta s t m 3169:: k a n)$ by expression of intact STM3169 from a plasmid. These findings provide the first evidence that STM3169 functions as a virulence factor of $S$. Typhimurium in a mouse infection model.

Because it is believed that intracellular Salmonella is likely to be restricted to the acquisition of nutrient substrates from infected host cells, the stringent response 


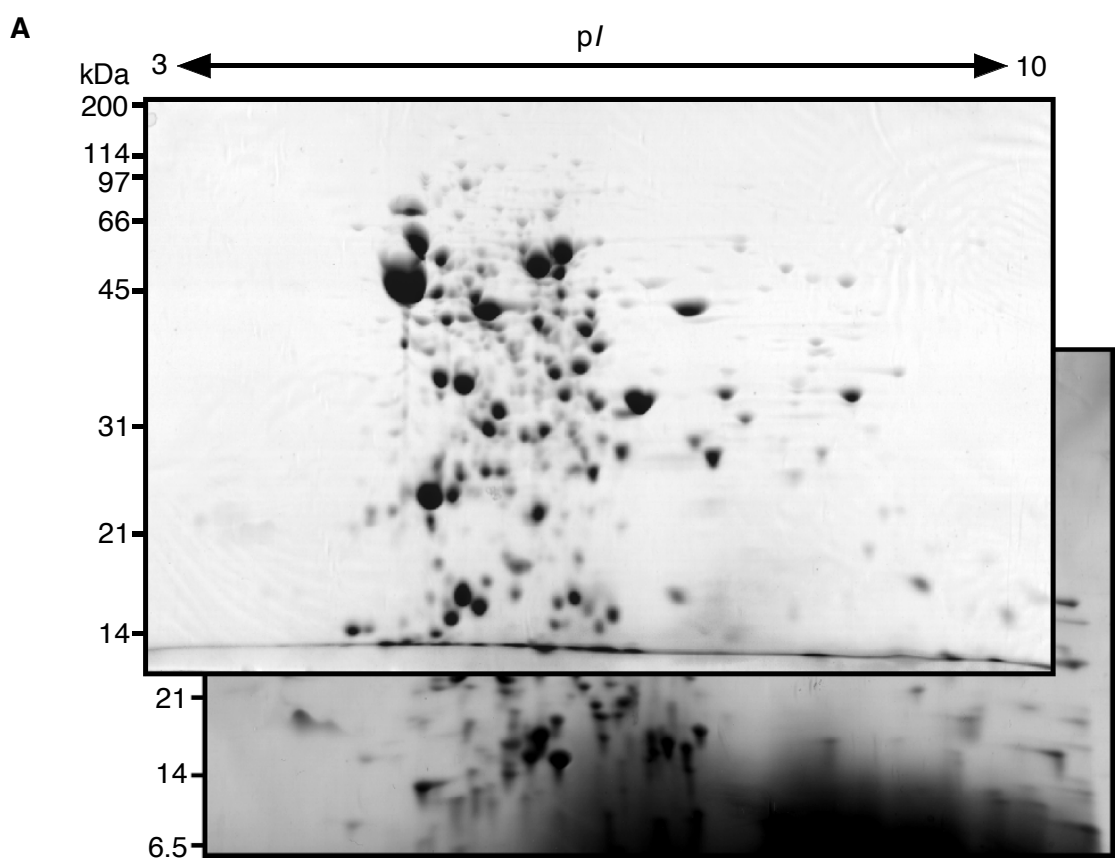

B

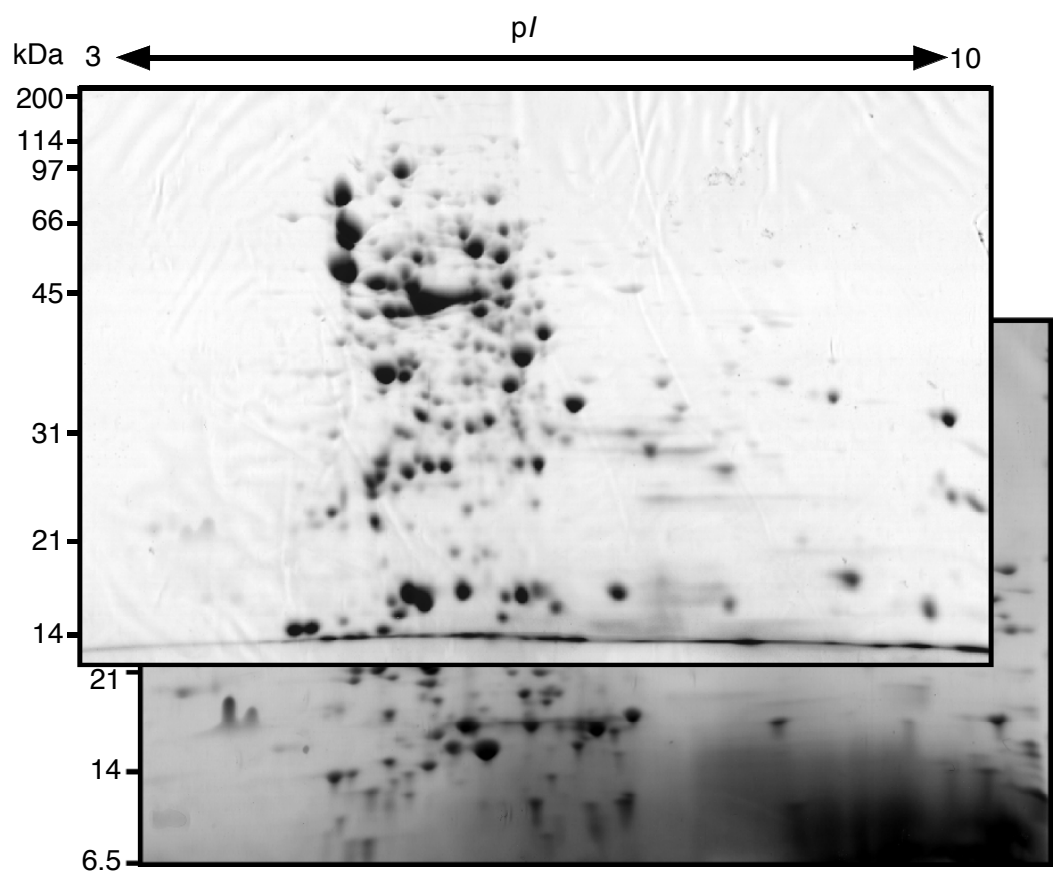

Figure 3 Comparison of the agarose 2-DE maps of S. Typhimurium wild-type SH100 (A) and ppGpp-deficient strain TM157 (B) during amino acid starvation. Both strains were grown under the same condition as described in Figure 1. Gels were stained with Coomassie Brilliant Blue.

could occur in SCV. Thus, we next analyzed the contribution of STM3169 to intracellular survival of S. Typhimurium in macrophages. In accordance with previous data that a $\mathrm{ppGpp}^{0}$ mutant strain deficient in both spoT and $\mathrm{rel} A$ genes resulted in a severe reduction of intracellular proliferation and suvival [12]. In contrast to the wild-type level of invasion, intracellular survival of
TH973 in RAW264.7 cells was reduced, compared with that of the wild-type strain. The reduced CFU of TH937 in IFN- $\gamma$ treated-RAW264.7 cells was not more severe than that in the $\Delta$ relA $\triangle$ spoT double mutant, $\Delta s s a V$ (SH113, SPI-2 T3SS component-defected mutant), and $\Delta s s r B$ (YY1, SPI-2 regulator mutant) strain, but was equal to that in the $\Delta s s e F$ (TM548, SPI-2 effector 
Table $1 \mathrm{~S}$. Typhimurium proteins regulated by ppGpp

\begin{tabular}{|c|c|c|c|c|c|}
\hline spot no. & STM no. & Gene & Fold & Anova $(p)$ & Average fold change determined by qRT-PCR \\
\hline \multicolumn{6}{|c|}{ Proteins expressed lower in $\Delta r e l A \Delta s p o T$ strain } \\
\hline 002,091 & STM2884 & $\operatorname{sip} C$ & 0.1 & 0.006 & $N D^{a}$ \\
\hline 005 & STM0781 & $\bmod A$ & 0.3 & 0.032 & $0.67 \pm 0.22$ \\
\hline 012 & STM3169 & Stm3169 & 0.3 & 0.004 & $0.18 \pm 0.01^{c}$ \\
\hline 014,213 & STM1796 & treA & 0.7 & 0.002 & $E C^{b}$ \\
\hline 015 & STM4403 & $c p d B$ & 0.6 & 0.011 & $0.25 \pm 0.06^{c}$ \\
\hline 027 & STM1954 & fliY & 0.5 & 0.033 & ND \\
\hline 028 & STM2884 & $\operatorname{sip} C$ & 0.1 & 0.009 & ND \\
\hline 029 & STM3557 & $u g p B$ & 0.4 & 0.019 & EC \\
\hline 029-2 & STM0748 & tolB & 0.4 & 0.019 & $0.25 \pm 0.03^{c}$ \\
\hline 037 & STM0209 & htrA & 0.6 & 0.032 & $0.60 \pm 0.35$ \\
\hline 040 & STM2638 & rseb & 0.3 & 0.011 & $0.88 \pm 0.35$ \\
\hline $040-2$ & STM1478 & $y d g H$ & 0.3 & 0.011 & $0.17 \pm 0.06^{c}$ \\
\hline 041 & STM1375 & $y n h G$ & 0.3 & 0.011 & EC \\
\hline 056 & STM1746 & oppA & 0.6 & 0.001 & $0.15 \pm 0.05^{c}$ \\
\hline 058 & STM1746 & oppA & 0.5 & 0.006 & $0.15 \pm 0.05^{c}$ \\
\hline 059 & STM1849 & yliB & 0.4 & 0.027 & EC \\
\hline 060 & STM3557 & ugp B & 0.3 & 0.006 & EC \\
\hline 062 & STM1091 & sop $B$ & 0.2 & 0.036 & ND \\
\hline 064 & STM4319 & phoN & 0.1 & 0.014 & $0.54 \pm 0.22$ \\
\hline 108 & STM0435 & yajQ & 0.5 & 0.038 & $0.12 \pm 0.05^{c}$ \\
\hline $108-2$ & STM1440 & sodCl & 0.5 & 0.038 & ND \\
\hline 153 & STM3318 & $y h b N$ & 0.6 & 0.047 & $0.28 \pm 0.12^{c}$ \\
\hline 154 & STM4405 & $y(f)$ & 0.2 & 0.049 & $0.30 \pm 0.02^{c}$ \\
\hline 184 & STM3348 & $\operatorname{deg} Q$ & 0.4 & 0.038 & EC \\
\hline 194 & STM1720 & $y c i O$ & 0.3 & 0.028 & $14.22 \pm 2.22^{c}$ \\
\hline \multicolumn{6}{|c|}{ Proteins expressed higher in $\triangle r e l A \Delta s p o T$ strain } \\
\hline 004 & STM3359 & mdh & 2.0 & 0.021 & ND \\
\hline 006 & STM3069 & $p g k$ & 1.4 & 0.037 & ND \\
\hline 008 & STM2681 & grpE & 1.5 & 0.018 & ND \\
\hline 068 & STM3342 & $\operatorname{ssp} A$ & 1.7 & 0.014 & EC \\
\hline 081 & STM2952 & eno & 1.7 & 0.014 & ND \\
\hline 096 & STM1700 & $f a b l$ & 1.8 & 0.041 & ND \\
\hline 098 & STM0232 & $a c c A$ & 2.2 & 0.017 & ND \\
\hline 101 & STM3446 & fusA & 3.7 & 0.022 & ND \\
\hline 109 & STM4055 & sodA & 2.0 & 0.044 & EC \\
\hline 115 & STM3415 & rpoA & 1.5 & 0.043 & EC \\
\hline 116 & STM4184 & aceA & 1.6 & 0.007 & ND \\
\hline 118 & STM0737 & $S U C B$ & 1.7 & 0.006 & ND \\
\hline 119 & STM2660 & $C l p B$ & 3.7 & 0.035 & ND \\
\hline 135 & STM0735 & $\operatorname{sdh} B$ & 2.1 & 0.002 & ND \\
\hline 142 & STM3063 & rpiA & 1.8 & 0.022 & ND \\
\hline 145 & STM4190 & pepE & 1.5 & 0.003 & ND \\
\hline 155 & STM0734 & $\operatorname{sdh} A$ & 2.9 & 0.039 & ND \\
\hline 186 & STM3282 & pnp & 3.2 & 0.013 & ND \\
\hline 187 & STM3446 & fusA & 2.3 & 0.031 & ND \\
\hline 210 & STM1305 & astD & 1.8 & 0.007 & $E C$ \\
\hline 222 & STM3502 & $O m p R$ & 1.7 & 0.025 & ND \\
\hline 227 & STM2378 & $f a b B$ & 1.6 & 0.035 & ND \\
\hline 231 & STM1746 & oppA & 1.8 & 0.012 & ND \\
\hline
\end{tabular}

${ }^{\mathrm{a} N D}$, not determined.

${ }^{b} E C$, already identified as a ppGpp-regulated protein in E. coli by Traxler et al. [30].

${ }^{C}$ mRNA level was significantly different between wild type and the $\triangle$ relA $\triangle$ spo $T$ mutant. 


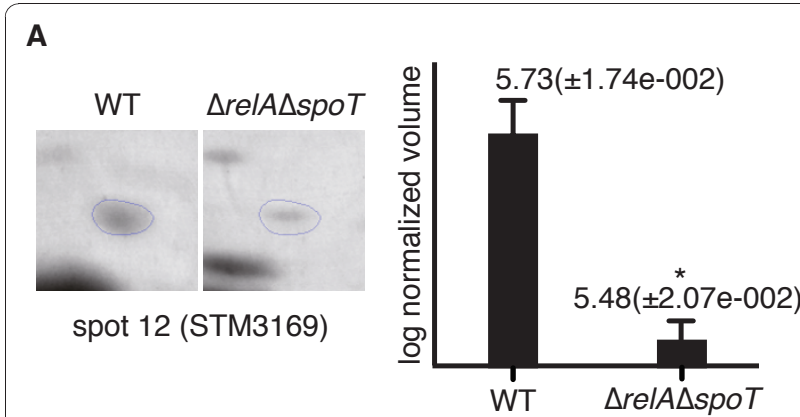

B

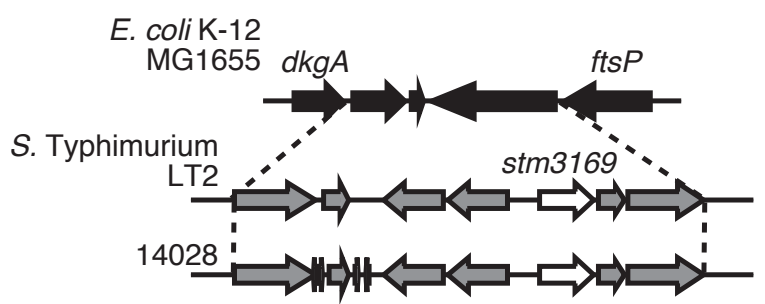

Figure 4 The S. Typhimurium-specific protein STM3169 is regulated by ppGpp in the stringent response. (A) Comparison of the STM3169 protein expression in the wild-type SH100 and $\triangle r e l A \Delta s p o T$ strain (TM157). An asterisk indicates that the difference was statistically significant $(P<0.05)$. (B) Genetic map of genes (open arrows) coding STM3169 within Salmonella-specific locus (gray arrows) and genes flanking the locus (closed arrows).

mutant) strain (Figure 5B and 5C). These results suggest that the expression of additional virulence factors, like STM3169, in macrophages might be affected in a highly avirulent phenotype of a ppGpp-deficient strain in mice.

\section{stm3169 is regulated by the SPI-2 transcriptional regulator ssr $B$}

It has been demonstrated that ppGpp mediates the expression of virulence-associated genes involved in bacterial invasion and intracellular growth and survival via global and/or gene-specific transcriptional regulators in $S$. Typhimurium $[12,14]$. Since intracellular growth and suvival of Salmonella in macrophages is dependent upon SPI-2 function, we next confirmed whether expression of stm3169 is regulated by the SsrAB twocomponent system, which positively controls the expression of SPI-2 genes as well as other genes belonging to the $\mathrm{SsrB}$ regulon [32]. To test this, we constructed S. Typhimurium strains carrying stm3169::lacZ transcriptional fusions on the chromosome in the wild-type (SH100) and $\Delta$ relA $\Delta$ spoT (TM157) genetic background. Salmonella strains carrying the stm3169::lacZ fusion gene (TH1162 and TH1164) were grown in defined MgM medium (pH 5.8) with $0.1 \%$ casamino acids and measured $\beta$-galactosidase activity. The transcription levels of stm3169::lacZ fusion were significantly

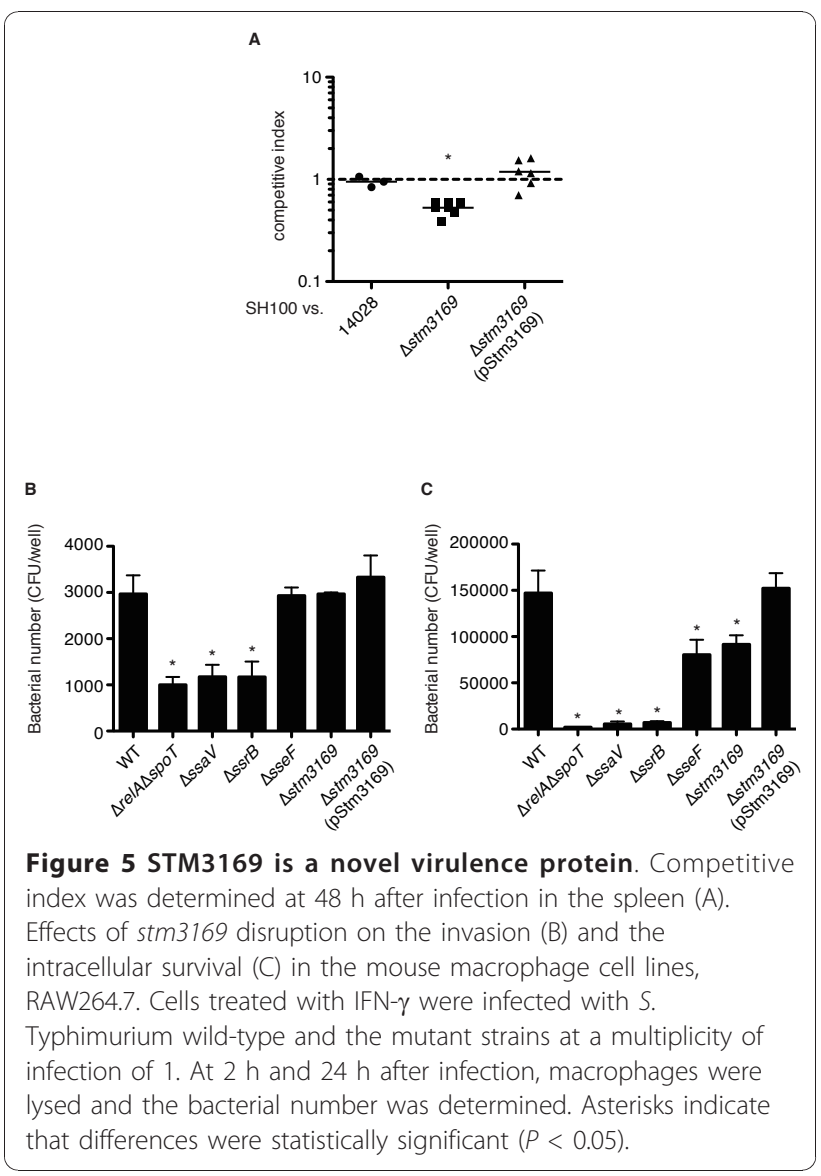

decreased in TM157 (Figure 6A). The reduced level was restored to the wild-type level by the introduction of an arabinose-inducible plasmid expressing $\mathrm{His}_{6}$-tagged RelA protein. We next transduced the $s s r B$ mutation ( $\Delta s s r B:: c a t)$ into a stm3169::lacZ fusion strain (TH1162). Strains carrying the stm3169::lacZ fusion gene with the $s s r B$ mutation were grown in MgM medium ( $\mathrm{pH} 5.8$ ), and $\beta$-galactosidase activity was measured. Control experiments were performed with the $s s a G:$ :lac $Z$ fusion gene (TM129). ssaG expression is strongly controlled by SsrB [33]. Similar to $s s a G:$ :lacZ, the transcription level of the stm3169::lacZ fusion gene was significantly decreased in strains carrying the $s s r B$ mutation (Figure 6B). Complementation was partially achieved for TM423 by expression of SsrB (SsrB-FLAG) on a plasmid (Figure $6 \mathrm{~B})$, probably due to the constitutive expression of SsrB from multi-copy-number palsmid pFLAG-CTC. Collectively, these data suggest that the novel virulence-associated factor STM3169 was regulated by the SPI-2 twocomponent regulatory system $\mathrm{SsrAB}$ as well as by ppGpp.

It has been reported that ppGpp regulates SPI-2encoded genes under aerobic condition [14]. To further characterize the transcriptional regulation of stm3169 by 


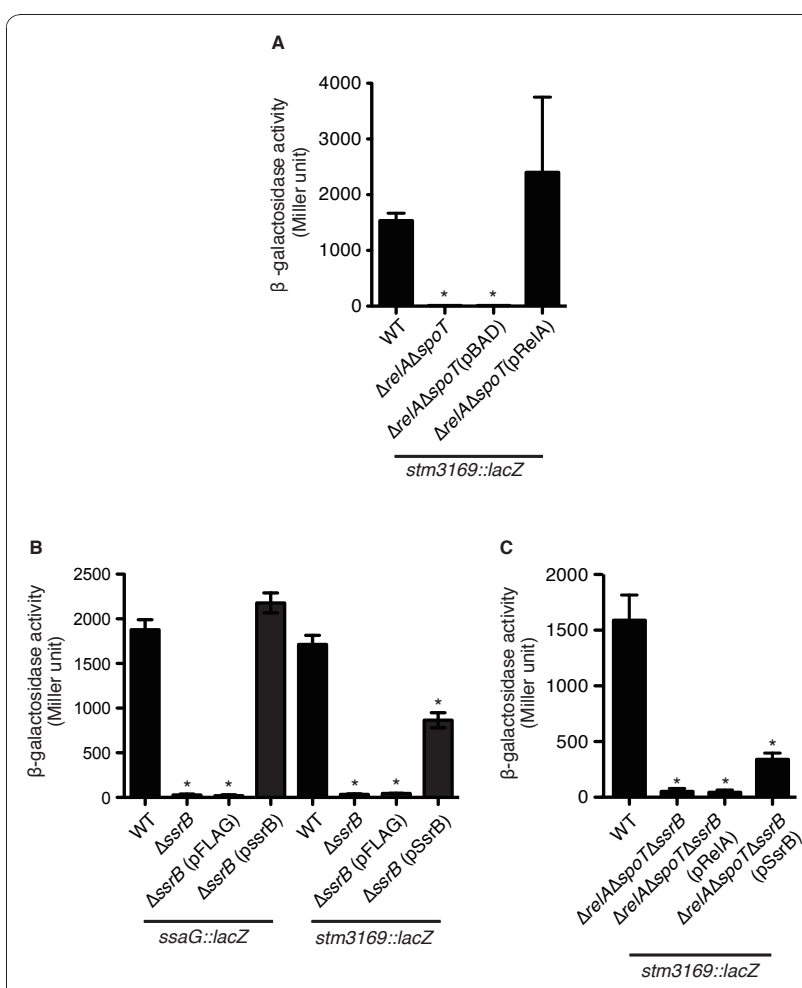

Figure 6 STM3169 is regulated by ppGpp and ssrB. Transcriptional activity of stm3169 in Salmonella muntant strains. Salmonella $\triangle r e l A \triangle s p o T(A), \triangle s s r B(B)$, and $\triangle r e l A \triangle s p o T \triangle s s r B(C)$ mutant strains carrying stm3196::/acZ fusion were incubated in $\mathrm{MgM}$ medium ( $\mathrm{pH} 5.8$ ) for $18 \mathrm{~h}$. The promoter activity of stm3169 was estimated by mesuring the $\beta$-garactosidase activity. L-arabinose (a final concentration of $0.001 \%$ ) and IPTG (a final concentration of $0.01 \mathrm{mM}$ ) were added in the medium for induction of RelA on pRelA and for SsrB on pSsrB, respectively. Asterisks indicate that differences were statistically significant $(P<0.05)$.

ppGpp and SsrB, we constructed a $\Delta$ relA $\Delta$ spoT $\Delta s s r B$ triple mutant strain (YY2), and examined the affect of the transcriptional activity on $s t m 3169:$ :lacZ fusion gene. While the transcriptional activity of $s t m 3169:$ :lacZ fusion in the triple mutant strain was significantly reduced at the same level of $\triangle r e l A \Delta s p o T$ double mutant strain, it could be restored by introduction of plasmid pSsrB expressing SsrB-FLAG but not pRelA expressing $\mathrm{His}_{6}{ }^{-}$ tagged RelA (Figure 6C). These results indicate that ppGpp is controlled the expression of stm3169 through SsrB.

STM3169 is homologous to DctP in Rhodobacter capsulatus with a $31 \%$ identity and a $73 \%$ similarity. DctP, along with DctQ and DctM, constitutes a tripartite ATP-independent periplasmic transporter (TRAP-T) system involved in succinate utilization, and DctP plays a role as an extracytoplasmic solute receptor in this transporter [34]. STM3170 and STM3171, which are located immediately downstream from STM3169, have a $66 \%$ and an $80 \%$ similarity with DctQ and DctM, respectively. These suggest that the TRAP-T in S. Typhimurium is composed of $\operatorname{stm} 3169$, stm3170, and stm3171 genes. In addition, two hypothetical operons, yiaOMN and stm4052-4054, are annotated as TRAP-T in the $S$. Typhimurium strain LT2 [31]. Recently, it has been reported that the TRAP-T (SiaPQM) in Haemophilus influenzae is essential for LPS sialylation and virulence [35]. Further research is necessary to determine the role of these transporters in $S$. Typhimurium virulence.

\section{Conclusions}

We constructed an agarose 2-DE reference map of amino-acid starved $S$. Typhimurium and identified a novel virulence-associated factor, STM3169, regulated by ppGpp by applying the map to comparative proteomics. stm3169 is also regulated by an SPI-2 two-component regulator, SsrB. Recently, it has been reported that the lack of ppGpp synthesis in Salmonella strains attenuates virulence and induces immune responses in mice [36]. Thus, further analysis of proteins regulated by ppGpp may lead to the development of new vaccines.

\section{Methods}

\section{Bacterial strains, primers, and culture conditions}

The bacterial strains and plasmids used in this study are listed in Table 2. The oligonucleotide primers used are listed in Table 3. Bacteria were grown in Luria-Bertani (LB) medium or on LB agar under conditions suitable for selection for resistance to ampicillin $(100 \mu \mathrm{g} / \mathrm{mL})$, chloramphenicol $(25 \mu \mathrm{g} / \mathrm{mL})$, nalidixic acid $(50 \mu \mathrm{g} / \mathrm{mL})$, or spectinomycin $(50 \mu \mathrm{g} / \mathrm{mL})$, as appropriate. To induce the bacterial stringent response, serine hydroxamate (Sigma; 0.005\%), an inhibitor of serine tRNA synthetase, was added to a $12 \mathrm{~h}$ culture in LB broth, and the bacteria were further incubated for $1 \mathrm{~h}$ [26]. Magnesium minimal medium ( $\mathrm{MgM}, \mathrm{pH} 5.8)$ was used to induce SPI-2 gene expression [6].

\section{Construction of mutants}

Nonpolar mutants of relA and spoT were constructed by allele exchange using the temperature- and sucrose-sensitive suicide vector pCACTUS [37]. The relA and spoT genes were amplified by PCR with the following primers: (1) relA-FW and relA-RV for relA and (2) spoTFW and spoT-RV for spoT. S. Typhimurium strain SH100 genomic DNA was used as the template. The PCR products were cloned into TA cloning vector pGEM-T Easy (Promega) generating plasmid pGEMrelA and pGEM-spoT, respectively. A disruption mutation of relA was created by the insertion of the HincIIdigested promoterless cat gene into a unique NruI site in the coding sequence of relA on pGEM-relA. The 
Table 2 Bacterial strains and plasmids used

\begin{tabular}{|c|c|c|}
\hline Strains & Relevant characteristics & Source/Ref. \\
\hline \multicolumn{3}{|c|}{$\begin{array}{l}\text { Bacterial strains } \\
\text { S. Typhimurium }\end{array}$} \\
\hline 14028 & wild-type & ATCC \\
\hline $\mathrm{SH} 100$ & Spontaneous nalidixic acid resistant derivative of wild-type 14028 & [44] \\
\hline TM157 & SH100 $\Delta$ relA::cat $\triangle$ spoT::kan & this study \\
\hline YY2 & SH100 $\Delta$ relA:::cat $\triangle$ spoT::kan $\triangle$ ssrB::tet & this study \\
\hline TH973 & $\mathrm{SH} 100 \Delta \operatorname{stm} 3169:: k a n$ & this study \\
\hline TH1162 & SH100 stm3169::lacZ & this study \\
\hline TH1164 & TM157 stm3169:IacZ & this study \\
\hline YY3 & TH1164 $\triangle$ ssrB::tet & this study \\
\hline TM129 & SH100 ssaG::IacZ & this study \\
\hline YY1 & SH100 $\Delta s s r B:: t e t$ & this study \\
\hline $\mathrm{SH} 113$ & SH100 $\Delta s s a V:: c a t$ & [11] \\
\hline TM548 & SH100 $\Delta s s e F:: k a n$ & this study \\
\hline \multicolumn{3}{|l|}{ E. coli } \\
\hline $\mathrm{DH} 5 \alpha$ & K-12 recA1 endA1 gyrA96 thi-1 hsdR17 supE44 (lacXYA-argR)U169 deoR (80 dlac (lacZ)M15) & Invirtogen \\
\hline SM10גpir & thi-1 thr leu tonA lacY supE recA::RP4-2-Tc::Mu $\lambda$ pir & [45] \\
\hline \multicolumn{3}{|l|}{ Plasmids } \\
\hline pGEM-Teasy & TA cloning vector & Promega \\
\hline pMW118 & pSC101-based low copy number plasmid & Nippon Gene \\
\hline pACYC184 & p15A-based low copy number plasmid, tet template & New England Biolabs \\
\hline pFLAG-CTC & FLAG tag expression vector & Sigma \\
\hline $\mathrm{pLD}-\mathrm{lac} Z \Omega$ & Integrational plasmid with promoterless lacZ gene & [39] \\
\hline pBAD-HisA & Expression vector for His6 fusion protein & Invitrogen \\
\hline pMW-Stm3169 & stm3169 gene in pMW118 & this study \\
\hline pLD-stm3169Z & stm3169:IacZ operon fusion in pLD-lacZ $\Omega$ & this study \\
\hline pLD-ssaGZ & ssaG::/acZ operon fusion in pLD-lacZ $\Omega$ & this study \\
\hline pRelA & pBAD-HisA expressing relA gene & this study \\
\hline pSsrB & pFLAG-CTC expressing ssrB gene & this study \\
\hline pKD46 & Red recombinase expression plasmid & [41] \\
\hline pKD4 & kan cassette used for gene deletion & [41] \\
\hline
\end{tabular}

Table 3 Primers

\begin{tabular}{|c|c|}
\hline Name & Nucleotide sequence $\left(5^{\prime} \text { to } 3^{\prime}\right)^{a}$ \\
\hline \multicolumn{2}{|c|}{ Construction of the deletion mutants } \\
\hline relA-FW & CGCCATCCCGCAATGGTITACATAA \\
\hline relA-RV & TCATTGTTCTGGCCATAACAGC \\
\hline spoT-FW & CTTGAAAACCATCATTCGCGCTGAACG \\
\hline spoT-RV & TCTGCGGTACGAATGATTGCAGAAACG \\
\hline stm3169-red-FW & ACGTTCATTCACAACATCAGCGGTATTACTGGCCGGCTGTGTGTAGGCTGGAGCTGCTTC \\
\hline stm3169-red-RW & ACATATTCTCGATGTATTCCAGATCCTTCGCTGACTGAGCCATATGAATATCCTCCTTAG \\
\hline sseF-red-FW & AACAGAACGAAATATGAAAATTCATATTCCGTCAGCGGCAGTGTAGGCTGGAGCTGCTTC \\
\hline sseF-red-RW & TGTCCATTAATGCAGGTGTAGTAGCAGATTGACAGAGCGCCATATGAATATCCTCCTTAG \\
\hline pAC-tet-FW & TTGGTAGCTCAGAGAACCTTCGAAAAACCG \\
\hline pAC-tet-RV & TCGCTCGCGTATCGGTGATTCATTCGCTA \\
\hline \multicolumn{2}{|c|}{ Construction of plasmids for the complementations } \\
\hline relA-FW2 & AGGCTCGAGGTCGCGGTAAGAAGT \\
\hline relA-RV2 & ACAAGCTTACTGTCTGGGGTITAC \\
\hline ssrB-FW & GGGCTCGAGGAATATAAGATCTTATTAGTA \\
\hline
\end{tabular}


Table 3 Primers (Continued)

\begin{tabular}{|c|c|}
\hline ssrB-RV & CCCGGATCCATACTCTATTAACCTCATTCT \\
\hline stm3169-FW & CCGCTCGAGAACACACGTTCATTCACAACATCAG \\
\hline stm3169-RV & GGAAGATCTATTCTCGATGTATTCCAGATCCTTC \\
\hline \multicolumn{2}{|c|}{ Construcion of the lacZ fusions } \\
\hline ssaG-Pro-FW & AAAGTCGACCAAATGCTCAGGTAGGAGGGC \\
\hline ssaG-Pro-RV & AAAGGATCCATCATCGATTCTGGGTTGAGC \\
\hline stm3169-Pro-FW & ACGCGTCGACGACGATTAAGCCGGTATGAAAATCA \\
\hline stm3169-Pro-RV & CGGGATCCTTACATATTCTCGATGTATTCCAGA \\
\hline \multicolumn{2}{|c|}{ Comfirmation of gene expression by qRT-PCR } \\
\hline gyrA-FW & AAGAGCTCCTATCTGGATTATGC \\
\hline gyrA-RV & TATTTACCGATTACGTCACCAAC \\
\hline relA-FW & ATTGTGCCATTCACCTATCAGTT \\
\hline relA-RV & GATATITITGTCACGATCCTGCT \\
\hline invF-FW & ATCGCTGCTGAATAGTGTAGAAG \\
\hline invF-RV & CATTTGTCTGCCAATTGAATAAT \\
\hline stm0209-FW & CCTGAACGTAGAAAATTACGAGA \\
\hline stm0209-RV & GTCAGGTTTTTCACCATGTTACT \\
\hline stm0435-FW & GTCAATCAGTTGCTCGATATTCTG \\
\hline stm0435-RV & TTAAATCAGCTTGACGATTTTCTTC \\
\hline stm0748-FW & TGAACCTGTACGTTATGGATCTC \\
\hline stm0748-RV & CGCCGTTAATGTTCATTTTATAC \\
\hline stm0781-FW & GAAGGCAAGATCACCGTATTT \\
\hline stm0781-RV & CTGATCAGCAGAGATGAAGAGAT \\
\hline stm1478-FW & ACAAAAGTTGAGGAGCTGAATAAAG \\
\hline stm1478-RV & GCCACTGACGCGTAATATGATAA \\
\hline stm1720-FW & TTGGTTGTAAAATTGAAGACAAAGG \\
\hline stm1720-RV & GTCCCCTTCAGGATAAAGGTGTAAT \\
\hline stm1746-FW & CGAATTATTCCAGAAACTGAAGAAA \\
\hline stm1746-RV & ATCGCCCTGATITTAACCTTATTA \\
\hline stm2638-FW & TATTCTGACGGTCTGTTTAGCTTTT \\
\hline stm2638-RV & GTACTGCCCTGAATTTGATACTGTC \\
\hline stm3169-FW & GTTACCAGAATAATGTCGCAGCTAT \\
\hline stm3169-RV & AATCATCCACATAAAAAGAATCTGG \\
\hline stm3318-FW & CAAACTCAGCCTTAATCTTATGC \\
\hline stm3318-RV & ACTITATCGGCGTTGATCTTAAT \\
\hline stm4319-FW & ATTAGTATTATCCGAGGCCAGAC \\
\hline stm4319-RV & CAGTCTTGCAAACTCTACTGCTC \\
\hline stm4403-FW & ATTGATATTCACAGCAACAAACC \\
\hline stm4403-RV & AGGTCAGGTTITTAATACGTTCC \\
\hline stm4405-FW & CGAACTGACGTTGAATAAAGATGAG \\
\hline stm4405-RV & AATTGTGGTCGTCTGGTATCTGT \\
\hline
\end{tabular}

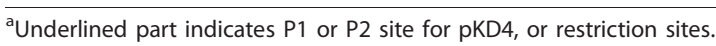

resulting plasmid pGEM-relA::cat was digested with BglII and then self-ligated, yielding plasmid pGEM$\Delta$ relA::cat. In contrast, the spoT gene was disrupted by the insertion of a SmaI-digested $\mathrm{Km}^{\mathrm{r}}$-encoding gene (kan) cassette from pUC18K [38] into NruI sites in the coding sequence of spoT on pGEM-spoT, thus generating pGEM- $\Delta$ spoT::kan. The disrupted gene was then subcloned using SalI and SphI into similarly digested
pCACTUS, and the resulting plasmid was introduced into strain $\mathrm{SH} 100$ by electroporation for allele exchange mutagenesis, which was carried out as described previously [39]. $\triangle$ relA $\triangle$ spoT mutant strain was created by phage P22-mediated transduction [40].

The PCR-based $\lambda$ Red recombinase system using pKD46 and pKD4 was performed to disrupt stm3169 or sseF [41]. The growth rate of these mutant strains in LB 
and $\mathrm{MgM}$ (pH5.8) broth showed the same levels to wild-type strain.

To construct $\Delta$ relA $\Delta$ spoT $\Delta s s r B$ mutant strain, the cloned $s s r B$ gene was disrupted by the insertion of a Tet ${ }^{r}$ encoding gene (tet) cassette, which was amplified with pAC-tet-FW and pAC-tet-RV primers using pACYC184 (New England Biolabs) as template. The $\Delta s s r B:$ tet fragment was amplified by PCR using ssrB-FW and ssrB-RV primers, and the resulting PCR product was introduced into $S$. Typhimurium SH100 carrying pKD46. The disrupted genes were transferred by phage P22 transduction into $\triangle$ relA $\triangle$ spoT mutant strain TM157.

To construct ssaG::lacZ and stm3169::lacZ transcriptional fusions, pLD-ssaGZ and pLD-stm3169Z were transferred from Escherichia coli SM10גpir to S. Typhimurium SH100 by conjugation. The fusions were introduced into SH100, $\Delta$ relA $\Delta$ spoT (TM157), $\Delta s s r B:: t e t$ (YY3), and $\Delta s s a V$ (SH113) mutant strains by phage P22mediated transduction. All constructs were verified by PCR or DNA sequencing.

\section{Construction of plasmids}

For construction of the complementing plasmid, pMWStm3169, stm3169 gene was amplified by PCR with stm3169-FW and stm3169-RV primers. S. Typhimurium SH100 genomic DNA was used as the template. The PCR products were digested with BglII and XhoI, and cloned into the Bglll-XhoI site on pMW118 (Nippon Gene), generating plasmid pMW-Stm3169.

To construct pRelA and pSsrB, the target genes were amplified by PCR with the following primers: relA-FW2 and relA-RV2 for relA and ssrB-FW and ssrB-RV for $s s r B$. The PCR product containing relA was digested with XhoI-HindIII and cloned into the same sites on pBAD-HisA (Invitrogen). The PCR product containing $s s r B$ was digested with XhoI-BamHI and cloned into the same sites on pFLAG-CTC (Sigma). pRelA and pSsrB expressed $\mathrm{His}_{6}$-tagged RelA and SsrB-FLAG fusion protein, respectively.

To construct lac $Z$ transcriptional fusions, the DNA fragments containing (predicted) promoter regions of ssaG were amplified by PCR using the primers ssaG-Pro-FW and ssaG-Pro-RV, and those containing promoter regions of stm3169 were amplified using stm3169-Pro-FW and stm3169-Pro-RV. The PCR products digested with SalI and BamHI were ligated into the same sites of pLD-lacZ $\Omega$ [39].

\section{Sample preparation for agarose 2-DE}

Agarose 2-DE samples were prepared from amino-acid starved S. Typhimurium strain SH100, as well as relA and spoT double knockout strain TM157 ( $\triangle$ relA $\Delta$ spoT). The cell pellets were washed twice with cold phosphate- buffered saline (PBS) and dissolved in lysis buffer containing $5 \mathrm{M}$ urea, $1 \mathrm{M}$ thiourea, $0.05 \% \mathrm{w} / \mathrm{v} \beta$-mercaptoethanol, and one tablet of protein inhibitor (Complete Mini EDTA-free; Roche Diagnostics, Mannheim, Germany), which was dissolved in $10 \mathrm{~mL}$ of the solution. The lysates were centrifuged $(104,000 \times g, 20 \mathrm{~min}$, $4^{\circ} \mathrm{C}$ ) and the clear supernatant was used.

\section{Proteome analysis}

We performed proteome analysis according to the procedures of Oh-Ishi et al. [25] and Kuruma et al. [42]. An aliquot of 200-300 $\mu \mathrm{L}$ (containing $500 \mu \mathrm{g}$ of protein) of sample solution was subjected to first-dimension IEF at $667 \mathrm{~V}$ for $18 \mathrm{~h}$ at $4^{\circ} \mathrm{C}$, followed by second-dimension SDS-PAGE. The slab gel was stained with CBB R-350 (PhastGel Blue R; GE Healthcare).

Protein spots were excised from a destained gel with $50 \%(\mathrm{v} / \mathrm{v}) \mathrm{ACN}$ and dried under vacuum. The proteins were digested in the gel with trypsin. Digested fragments of 15 pmol were loaded on a Liquid Chromatography-Mass Spectrometry/Mass Spectrometry (LCMS/MS), which consisted of Nanospace SI-2 (Shiseido Fine Chemicals), an HPLC (LCQ Deca), and an ion trap mass spectrometer (Thermo Finnigan). We identified a protein from measured masses of the tryptic peptides and their MS/MS fragments using the SEQUEST program. When the top-ranked candidates had SEQUEST scores lower than 90, we inspected the raw MS and MS/MS spectra of peptides to judge their qualities. We classified identified proteins according to the Kyoto Encyclopedia of Genes and Genomes (KEGG) PATHWAY database http://www.genome.ad. jp/kegg/pathway.html.

Gel-to-gel comparisons between SH100 and TM153 were performed for two separately prepared samples. Each scanned 2-DE gel image was analyzed with the gel image analysis software SameSpots (Progenesis).

\section{RNA extraction and quantitative real-time $\mathrm{PCR}$}

$S$. Typhimurium strains were grown in LB and ppGpp expression was induced as described above. Total RNA was isolated from the bacterial culture using RNAprotect Bacteria Reagent and the RNeasy Protect Bacteria Mini Kit with the gDNA Eliminator spin column (Qiagen) according to the manufacturer's instructions. cDNA was synthesized using the QuantiTect Reverse Transcription Kit (Qiagen). Real-time PCR was performed with the primer pairs listed in Table 3 using QuantiTect SYBR Green and the 7900HT Sequence Detection System (Applied Biosystems). The data were analyzed using the comparative Ct method (Applied Biosystems). Transcription of the target gene was normalized to the levels of $g y r A$ mRNA. 


\section{Mouse infections}

For the competitive index assay, female BALB/c mice (5-6 weeks old) were used for the mouse infection study and were housed at Kitasato University according to the standard Laboratory Animal Care Advisory Committee guidelines. Mice were inoculated by intraperitoneal infection with $100 \mu \mathrm{L}$ of inoculum containing a total of $1 \times 10^{5}$ bacteria (each strain at $5 \times 10^{4}$ ), consisting of an equal number of wild-type and mutant strains. At $48 \mathrm{~h}$ after infection, the mice were sacrificed by carbon dioxide inhalation. The spleens were homogenized in cold PBS by mechanical disruption. The number of each strain in the spleen was determined by plating a dilution series of the lysate onto LB agar alone and LB agar with appropriate antibiotics. Each competitive index value was calculated as [mutant/wild-type] output/[mutant/wild-type] input and represented as the mean of at least three independent infections.

\section{Macrophage survival assay}

Cells of a mouse macrophage-like line, RAW264.7, were diluted in DMEM containing 10\% FBS and seeded in 24-well plates at a density of $5 \times 10^{5}$ cells per well. $S$. Typhimurium strains were used to infect RAW264.7 cells at a multiplicity of infection of 1 . The bacteria were centrifuged onto the cells $(500 \times g, 5$ min) and incubated for $25 \mathrm{~min}$ at $37^{\circ} \mathrm{C}$ in a $5 \% \mathrm{CO}_{2}$ incubator. Cells were washed three times with PBS, and DMEM containing interferon- $\gamma$ (IFN- $\gamma$ ) (100 units/ well; Peprotech) and gentamicin $(100 \mu \mathrm{g} / \mathrm{mL}$; Sigma $)$ was added. After 95 min of incubation, the medium was replaced with DMEM containing IFN- $\gamma$ (100 units/ well) and gentamicin $(10 \mu \mathrm{g} / \mathrm{mL})$. The number of intracellular bacteria was determined at $2 \mathrm{~h}$ and $24 \mathrm{~h}$ after infection. For the enumeration of intracellular bacteria, the cells were washed three times with PBS and lysed in $1 \%$ Triton X-100, and bacteria were quantified by spreading serial 10-fold dilutions of RAW264.7 cell lysates on LB agar plates to count the colony-forming units (CFU). Each experiment was repeated three times.

\section{$\beta$-galactosidase assay}

$\beta$-galactosidase activities of reporter gene fusions were determined according to a standard procedure [43].

\section{Statistical analysis}

The competitive index, mRNA expression, and bacterial proliferation in macrophage cells were compared using Student's t-test. For comparative proteomics, the intensity of the spot was compared by one-way ANOVA. Values of $P<0.05$ were considered statistically significant.

\section{Additional material}

Additional file 1: Table S1. Proteins identified on the reference map.

\section{Acknowledgements}

We thank Toru Hattori (SCRUM inc, Japan) for 2-DE gel image analysis. We thank Kaori Dobashi, Nobue Nameki, Masato Hosono, Kohei Yamashita, and Ayako Mizuta for their technical assistance.

This work was supported in part by Grants-in-Aid for Young Scientists (B) (17790291 and 22790415 for TH) and for Scientific Research (C) (17590398 and 21590490 for NO) from the Ministry of Education, Culture, Sports, Science, and Technology of Japan, and by a Kitasato University Research Grant for Young Researchers (2010 for TH).

\section{Author details}

'Department of Microbiology, School of Pharmacy, Kitasato University, 5-9-1 Shirokane, Minato-ku, Tokyo 108-8641, Japan. ${ }^{2}$ Laboratory of Biomolecular Dynamics, Department of Physics, Kitasato University School of Science, 115-1 Kitasato, Minami-ku, Sagamihara City, Kanagawa 252-0373, Japan.

\section{Authors' contributions}

$\mathrm{TH}, \mathrm{SM}, \mathrm{YYO}, \mathrm{YKO}$, and SSI performed the experiments. TH and $\mathrm{NO}$ designed the experiments. TMi constructed the TM157, TM129, and TM548 strains. YKu assisted with the experiments. MOI, TMa, and HD advised regarding the design of the experiments. $\mathrm{TH}$ and $\mathrm{NO}$ wrote the paper.

Received: 1 September 2010 Accepted: 21 December 2010

Published: 21 December 2010

\section{References}

1. Galan JE, Curtiss R: Cloning and molecular characterization of genes whose products allow Salmonella typhimurium to penetrate tissue culture cells. Proc Natl Acad Sci USA 1989, 86(16):6383-6387.

2. Lundberg U, Vinatzer U, Berdnik D, von Gabain A, Baccarini M: Growth phase-regulated induction of Salmonella-induced macrophage apoptosis correlates with transient expression of SPI-1 genes. J Bacteriol 1999, 181(11):3433-3437.

3. Shea JE, Hensel M, Gleeson C, Holden DW: Identification of a virulence locus encoding a second type III secretion system in Salmonella typhimurium. Proc Natl Acad Sci USA 1996, 93(6):2593-2597.

4. Ochman H, Soncini FC, Solomon F, Groisman EA: Identification of a pathogenicity island required for Salmonella survival in host cells. Proc Natl Acad Sci USA 1996, 93(15):7800-7804.

5. Steele-Mortimer O, Brumell JH, Knodler LA, Meresse S, Lopez A, Finlay BB: The invasion-associated type III secretion system of Salmonella enterica serovar Typhimurium is necessary for intracellular proliferation and vacuole biogenesis in epithelial cells. Cell Microbiol 2002, 4(1):43-54.

6. Beuzon CR, Banks G, Deiwick J, Hensel M, Holden DW: pH-dependent secretion of SseB, a product of the SPI-2 type III secretion system of Salmonella typhimurium. Mol Microbiol 1999, 33(4):806-816.

7. Bijlsma JJ, Groisman EA: The PhoP/PhoQ system controls the intramacrophage type three secretion system of Salmonella enterica. Mol Microbiol 2005, 57(1):85-96.

8. Lee AK, Detweiler CS, Falkow S: OmpR regulates the two-component system SsrA-ssrB in Salmonella pathogenicity island 2. J Bacteriol 2000, 182(3):771-781.

9. Linehan SA, Rytkonen A, Yu XJ, Liu M, Holden DW: SlyA regulates function of Salmonella pathogenicity island 2 (SPI-2) and expression of SPI-2associated genes. Infect Immun 2005, 73(7):4354-4362.

10. Navarre WW, Halsey TA, Walthers D, Frye J, McClelland M, Potter JL, Kenney LJ, Gunn JS, Fang FC, Libby SJ: Co-regulation of Salmonella enterica genes required for virulence and resistance to antimicrobial peptides by SlyA and PhoP/PhoQ. Mol Microbiol 2005, 56(2):492-508.

11. Okada N, Oi Y, Takeda-Shitaka M, Kanou K, Umeyama H, Haneda T, Miki T, Hosoya S, Danbara H: Identification of amino acid residues of Salmonella SlyA that are critical for transcriptional regulation. Microbiology 2007, 153(Pt 2):548-560. 
12. Pizarro-Cerda J, Tedin K: The bacterial signal molecule, ppGpp, regulates Salmonella virulence gene expression. Mol Microbiol 2004, 52(6):1827-1844

13. Song M, Kim HJ, Kim EY, Shin M, Lee HC, Hong Y, Rhee JH, Yoon H, Ryu S, Lim S, et al: ppGpp-dependent stationary phase induction of genes on Salmonella pathogenicity island 1.J Biol Chem 2004, 279(33):34183-34190.

14. Thompson A, Rolfe MD, Lucchini S, Schwerk P, Hinton JC, Tedin K: The bacterial signal molecule, $\mathrm{ppGpp}$, mediates the environmental regulation of both the invasion and intracellular virulence gene programs of Salmonella. J Biol Chem 2006, 281(40):30112-30121.

15. Dalebroux ZD, Svensson SL, Gaynor EC, Swanson MS: ppGpp conjures bacterial virulence. Microbiol Mol Biol Rev 2010, 74(2):171-199.

16. Cashel M, Gentry DR, Hernandez DR, Vinella D: The stringent response. Escherichia coli and Salmonella typhimurium: Cellular and Molecular Biology, 21996.

17. Magnusson LU, Farewell A, Nystrom T: ppGpp: a global regulator in Escherichia coli. Trends Microbiol 2005, 13(5):236-242.

18. Paul BJ, Ross W, Gaal T, Gourse RL: rRNA transcription in Escherichia coli. Annu Rev Genet 2004, 38:749-770.

19. Jishage M, Kvint K, Shingler V, Nystrom T: Regulation of sigma factor competition by the alarmone ppGpp. Genes Dev 2002, 16(10):1260-1270.

20. Braeken $K$, Moris M, Daniels R, Vanderleyden J, Michiels J: New horizons for (p)ppGpp in bacterial and plant physiology. Trends Microbiol 2006, 14(1):45-54.

21. Zhao G, Weatherspoon N, Kong W, Curtiss R, Shi Y: A dual-signal regulatory circuit activates transcription of a set of divergent operons in Salmonella typhimurium. Proc Natl Acad Sci USA 2008, 105(52):20924-20929.

22. Chevalier F: Highlights on the capacities of "Gel-based" proteomics. Proteome Sci 2010, 8:23.

23. Bae SH, Harris AG, Hains PG, Chen H, Garfin DE, Hazell SL, Paik YK, Walsh BJ, Cordwell SJ: Strategies for the enrichment and identification of basic proteins in proteome projects. Proteomics 2003, 3(5):569-579.

24. Oh-lshi M, Maeda T: Disease proteomics of high-molecular-mass proteins by two-dimensional gel electrophoresis with agarose gels in the first dimension (Agarose 2-DE). J Chromatogr B Analyt Technol Biomed Life Sci 2007, 849(1-2):211-222.

25. Oh-Ishi M, Satoh M, Maeda T: Preparative two-dimensional gel electrophoresis with agarose gels in the first dimension for high molecular mass proteins. Electrophoresis 2000, 21(9):1653-1669.

26. Tosa T, Pizer LI: Effect of serine hydroxamate on the growth of Escherichia coli. J Bacteriol 1971, 106(3):966-971.

27. Jarvik T, Smillie C, Groisman EA, Ochman H: Short-term signatures of evolutionary change in the Salmonella enterica serovar Typhimurium 14028 genome. J Bacteriol 2010, 192(2):560-567.

28. Jing HB, Yuan J, Wang J, Yuan Y, Zhu L, Liu XK, Zheng YL, Wei KH, Zhang XM, Geng HR, et al: Proteome analysis of Streptococcus suis serotype 2. Proteomics 2008, 8(2):333-349.

29. Ying T, Wang H, Li M, Wang J, Shi Z, Feng E, Liu X, Su G, Wei K, Zhang X, et al: Immunoproteomics of outer membrane proteins and extracellula proteins of Shigella flexneri 2a 2457T. Proteomics 2005, 5(18):4777-4793.

30. Traxler MF, Summers SM, Nguyen HT, Zacharia VM, Hightower GA, Smith JT, Conway T: The global, ppGpp-mediated stringent response to amino acid starvation in Escherichia coli. Mol Microbiol 2008, 68(5):1128-1148.

31. McClelland M, Sanderson KE, Spieth J, Clifton SW, Latreille P, Courtney L, Porwollik S, Ali J, Dante M, Du F, et al: Complete genome sequence of Salmonella enterica serovar Typhimurium LT2. Nature 2001, 413(6858):852-856.

32. Worley MJ, Ching KH, Heffron F: Salmonella SsrB activates a global regulon of horizontally acquired genes. Mol Microbiol 2000, 36(3):749-761.

33. Walthers D, Carroll RK, Navarre WW, Libby SJ, Fang FC, Kenney L: The response regulator SsrB activates expression of diverse Salmonella pathogenicity island 2 promoters and counters silencing by the nucleoid-associated protein H-NS. Mol Microbiol 2007, 65(2):477-493.

34. Kelly DJ, Thomas GH: The tripartite ATP-independent periplasmic (TRAP) transporters of bacteria and archaea. FEMS Microbiol Rev 2001 25(4):405-424.

35. Jenkins GA, Figueira M, Kumar GA, Sweetman WA, Makepeace K, Pelton SI, Moxon R, Hood DW: Sialic acid mediated transcriptional modulation of a highly conserved sialometabolism gene cluster in Haemophilus influenzae and its effect on virulence. BMC Microbiol 10:48.
36. Na HS, Kim HJ, Lee HC, Hong Y, Rhee JH, Choy HE: Immune response induced by Salmonella typhimurium defective in ppGpp synthesis. Vaccine 2006, 24(12):2027-2034.

37. Morona R, van den Bosch L, Manning PA: Molecular, genetic, and topological characterization of O-antigen chain length regulation in Shigella flexneri. J Bacteriol 1995, 177(4):1059-1068.

38. Menard R, Sansonetti PJ, Parsot C: Nonpolar mutagenesis of the ipa genes defines IpaB, IpaC, and IpaD as effectors of Shigella flexneri entry into epithelial cells. J Bacteriol 1993, 175(18):5899-5906.

39. Miki T, Okada N, Danbara H: Two periplasmic disulfide oxidoreductases, DsbA and SrgA, target outer membrane protein SpiA, a component of the Salmonella pathogenicity island 2 type III secretion system. J Biol Chem 2004, 279(33):34631-34642.

40. Sternberg NL, Maurer R: Bacteriophage-mediated generalized transduction in Escherichia coli and Salmonella typhimurium. Methods Enzymol 1991, 204:18-43.

41. Datsenko KA, Wanner BL: One-step inactivation of chromosomal genes in Escherichia coli K-12 using PCR products. Proc Natl Acad Sci USA 2000, 97(12):6640-6645.

42. Kuruma H, Egawa S, Oh-lshi M, Kodera Y, Satoh M, Chen W, Okusa H, Matsumoto K, Maeda T, Baba S: High molecular mass proteome of androgen-independent prostate cancer. Proteomics 2005, 5(4):1097-1112.

43. Miller JH: A Short Course in Bacterial Genetics. Cold Spring Harbor Laboratory Press, Cold Spring Harbor, NY; 1992, 72-74.

44. Gotoh H, Okada N, Kim YG, Shiraishi K, Hirami N, Haneda T, Kurita A Kikuchi Y, Danbara H: Extracellular secretion of the virulence plasmidencoded ADP-ribosyltransferase SpvB in Salmonella. Microb Pathog 2003, 34(5):227-238

45. Simon R, Priefer U, Puhler A: A Braod host range mobilization system for in vivo genetic engineering: transposon mutagenesis in gram negative bacteria. Bio/Technology 1983, 1:784-791.

doi:10.1186/1471-2180-10-324

Cite this article as: Haneda et al: Comparative proteomic analysis of Salmonella enterica serovar Typhimurium ppGpp-deficient mutant to identify a novel virulence protein required for intracellular survival in macrophages. BMC Microbiology 2010 10:324.

\section{Submit your next manuscript to BioMed Central and take full advantage of:}

- Convenient online submission

- Thorough peer review

- No space constraints or color figure charges

- Immediate publication on acceptance

- Inclusion in PubMed, CAS, Scopus and Google Scholar

- Research which is freely available for redistribution 